\title{
A Novel Research on Incorporation of LPAR Routing Framework with Conventional Routing Networks in ODMRP
}

\author{
M. Chandramouli Reddy \\ Research Scholar, Dept. of CSE, \\ Mewar University, Rajasthan, India.
}

\author{
P. Venkata Krishna, PhD \\ Assistant Professor, SPMVV, \\ Andhra Pradesh, India
}

\begin{abstract}
Mobile Adhoc network are wireless adhoc networks and are considered as a popular concept in mobile wireless commutation and it is continuously self-configuring and is independent of any infrastructure. Location based point to point adaptive routing (LPAR) is prevalent for use in the mobile adhoc networks. The protocol which is employed here takes the help of a three state route discovery technique. This process is done in a point to point manner for optimized performance which includes the reduction of the reduction of the routing overhead along with the maximization of throughput when considering medium as well as large mobile adhoc networks. This paper has undertaken an in-depth study of the available methods of mobile adhoc network routing and proposes a unique technique for Incorporation of LPAR Routing Framework with Conventional Routing networks In ODMRP. Here, the said technique is implemented using the Cross-Layered Ant Colony Optimization Multicast Routing Protocol which is discussed in detail in the preceding sections. The study undertakes simulation in the MATLAB software package for the same with the help of mobile networks and the results have been discussed from the simulation so performed.
\end{abstract}

\section{Keywords}

MANET, ODMRP, LPAR, CLSODMRP, CLAMR, Ant Colony Optimization.

\section{INTRODUCTION}

\subsection{Mobile Adhoc Network (MANET)}

Mobile communication is inevitable in today's times. With advancement in communication technology, wireless communication with the help of networked devices is becoming highly popular. Wireless Adhoc Networks can be conceptualized as decentralized wireless networks which have the freedom from wired connections, access points and routers which are thought as the primary and conventional infrastructure for wireless communications (Karyotis, Stai, \&Papavassiliou, 2013).

Mobile Adhoc network known as the MANET hereafter are wireless adhoc networks and are considered as a popular concept in mobile wireless commutation and it is continuously self-configuring and is independent of any infrastructure. It can change its location at the same time configuring itself in the process. Hence, such networks can be established dynamically (Macker, 1999).

MANETs are widely employed when a mobile communication network is required for the collection of data from sensors or communication with the sensors for the same. They are a favorite choice when the area of application is related to data mining as well as monitoring (Goldsmith, \& Wicker, 2002).

\subsection{Location based point to point adaptive routing (LPAR)}

The above description of MANETS make it clear that the mobile adhoc networks are designed to have fundamentally dynamic nature. These networks usually consist of numerous nodes which are an integral part of the network. These nodes usually exhibit different mobility levels; as a result of which the topology of the network constantly changes and the MANET is found in various node configurations which are dynamically varying. MANETs are usually employed for the purpose of communication or data transfer. But with dynamically varying topology, there is no fixed path for the data to traverse form the source node to the destination node. Hence, the source node during the process of data transfer, may require to recalculate the route to the destination numerous times for the successful establishment of the channel for the data transfer thereby undertaking required transmission of the data.

This recalculation and determination of the routes dynamically and spontaneously over the network takes up significant part of the available bandwidth of the network. To address this, the traditional studies have proposed various techniques for the reactive route discovery. However, it has been found that these strategies which employ the concept of reactive route discovery which is based on the flooding theory are limited by the constraint of scalability and these are rendered obsolete with the increase in the number of nodes and eventually the size of the network (Tseng, Ni, Chen, \&Sheu, 2002).

Various studies have tried to address this problem (Perkins, Belding-Royer \& Das, 2003; Ko, \& Vaidya, 2000; Aggelou, \&Tafazolli, 1999) and reduce the prominent effect of the flooding effect which is significantly seen in the protocols involving source routing. The study undertaken by Abolhasan, Wysocki, \&Dutkiewicz (2003)can be considered as a significant contribution to the field of source routing for they have proposed a unique and novel approach to help the reduction of overheads in point to point routing.

In the field of point to point routing, the decision of routing can be made by each and every node which coincides along the path to destination. This is a much more adaptable strategy when considering the continuously changing topology of the network. With the help of this strategy, the recalculations at the source are reduced thereby reducing the bandwidth required for the purpose of routing. 
Location based point to point adaptive routing (LPAR) is popular for use in the mobile adhocnetworks. The protocol which is employed here takes the help of a three state route discovery technique. This process is done in a point to point manner for optimized performance which includes the reduction of the reduction of the routing overhead along with the maximization of throughput when considering medium as well as large mobile adhoc networks.

As explained earlier, the data transmission in the location based point to point adaptive routing is adaptable to the dynamically varying conditions of the network. To achieve this, simultaneous primary and secondary data forwarding technique is employed for the purpose of data transfer from source to destination. This is especially valid under the condition that the route is changed during the process of data transmission (Abolhasan, Wysocki, \&Dutkiewicz, 2003).

\subsection{On-Demand Multicast Routing Protocol (ODMRP)}

In the communication using mobile adhoc wireless networks multicast has emerged as a highly focussed research area. With the growth of communication technology, multicast is considered to be an inevitable aspect of internet and related applications. The concept of multicast deals with the delivery of data to several addresses or multiple nodes from a single source at the same time (Chlamtac, Conti, \& Liu, 2003).

To hold multicast in mobile adhoc networks which are dynamically changing environment of network nodes, the conventionally used static protocols do not perform well. Also, the tree structures developing for the purpose of holding multicast are relatively fragile and their continuous readjustment is inevitable in the cases of changing connectivity. Also inevitable and fundamental to a typical multicast tree is a global routing substructure like a distance vector or a link state (Layuan, \&Chunlin, 2007)

Considering the dynamic state of the adhoc networks, there is a frequent update in the link structure tables or the distance vector computation tables. This triggers the increase in the channel and processing overhead to a high value. Hence, the mobile networks which have a limited bandwidth and are constrained on power usually make the designing process of the multicast protocol, challenging (Boppana, \&Konduru, 2001).

A solution to the same is proposed in the study by Lee, Gerla, \& Chiang, (1999) in the form of On-Demand Multicast Routing Protocol (ODMRP). This technique is mesh based opposed to the conventional tree based schemes. The multicast in this technique is undertaken by the concept of forwarding group wherein the multicast packets are forwarderd by only a selected subset of nodes through scoped flooding technique. Moreover, on-demand procedures are applied for the dynamic building of routes and the maintenance of the membership to the multicast group. Studies have shown that ODMRP can be considered as a well suited option when discussing adhoc wireless networks having mobile hosts. Also, it is of great importance in cases where the MANET has a limiter chunk of available bandwidth and highly dynamic variation of topology as well as when the power is constrained (Lee, Gerla, \& Chiang, 1999).

\subsection{Cross Layered Secure On-Demand Multicast Routing Protocol (CLSODMRP)}

The previous section discusses the on-demand multicast routing protocol in which minimum hop count is preferred for the purpose of routing optimization. Here, all the nodes take the same path for the purpose of forwarding the multicast packets. Thus, the state of the nodes is loaded for a longer period of time. This results in congestion and increased overhead which eventually leads to an inefficient throughput of the network. Moreover, the technique is bind to the consequences when there is misbehavior of the nodes resulting from the assumption that all the nodes respond in a proper way. This is a threat to the security and reliability of the network.

To address the above two issues at the same time, the Cross Layered Secure On-Demand Multicast Routing Protocol is utilized wherein the throughput is maximized as well as the congestion and overhead is reduced along with providing required security to the network. This is undertaken by implementing cross layer ODMRP which solves the primary issue of throughput maximization and overhead reduction.

Cross layer ODMRP refers to the crossing of the prescribed parameters of the network layer as well as the MAC layer. The minimum hop count in this case is counted as the combination of network layer and MAC layer. Thereby, the measure of the cross layer is the product obtained from minimum hop count, node load level and available bandwidth (Jetcheva, \& Johnson, 2001).

\subsection{Cross-Layered Ant Colony Optimization Multicast Routing Protocol (CLAMR)}

The reduction in overheads in any MANET can be done by reducing the overall energy consumption of the network nodes. This usually comes as a challenging issue in the MANETs for all of these work on batteries and power interruption could lead to interruption of the network node. Thus, the technique of ant colony optimization is suggested for the purpose of routing in MANETs. This novel concept of routing with ant colony optimization uses special parameters in the fitness function definition so that the energy consumption in the network nodes can be reduced.

Cross-Layered Ant Colony Optimization Multicast Routing Protocol is inspired from the biology and the practices followed by colonies of ants while transferring food from source to the destination. This technique can be interpret as QoS enabled multipath routing protocol. This can be considered as an advancement of the conventional on-demand multicast routing protocol (ODMRP). Here, the basis of functioning is the mechanism explained in ODMRP and the resultant is an efficient protocol which reduces the overhead to a minimum value ( $\mathrm{Sim} \& \mathrm{Sun}$, 2003).

\section{PROBLEM STATEMENT}

The concept of ODMRP prefers that all the nodes take the same path for the purpose of forwarding the multicast packets. Thus, the state of the nodes is loaded for a longer period of time. This results in congestion and increased overhead which eventually leads to an inefficient 
throughput of the network. To address the issue, this paper proposes various enhanced ODMRP mechanisms for effectively transmitting data in Mobile Adhoc Networks such as CLSODMRP [Cross Layered Secure ODMRP] and CLAMR [Cross-Layered Ant Colony Optimization Multicast Routing Protocol] thereby helping in the improvement of quality of performance of mobile adhoc networks measured in terms of parameters like throughput, packet delivery ratio, end to end delay, packet loss, etc.

\section{PROPOSED SYSTEM}

The study undertaken proposes a Novel Research on Incorporation of LPAR Routing Framework with Conventional Routing networks In ODMRP

\subsection{Main Objectives:}

The study is proposed in the field of Mobile communication which has developed as an interesting research topic in recent era. The concept of MANET explained above, comes under the cellular network where internet like service is provided to number of users. Unlike cellular networks

MANETs are made up of a number of end-user nodes, which are proficient in determining routes to different parts of their networks, without using a base-station or a centralized administrator. Multicasting generally means transmission of packets from a source or a group of sources to a group of one or more hosts that are identified by a single destination address. Multicasting greatly reduces the transmission cost when sending the same packet to multiple recipients.

On Demand Multicast Routing Protocol (ODMRP) where used for routing in MANET which has an advantage to avoid channel overhead and improve scalability. As ODMRP prefers utilizing minimum hop count for routing optimization all the nodes choose the same route for forwarding the packets.

As a result the nodes stay in a state of load for prolonged periods of time causing congestion and overhead adversely affecting the throughput. In order to improve these drawbacks, we propose various enhanced ODMRP mechanisms like CLSODMRP [Cross Layered Secure ODMRP] and CLAMR [Cross-Layered Ant Colony Optimization Multicast Routing Protocol]for effectively transmitting data between nodes.

In conventional routing method, the link failure of active route and reduced route stability remains to be a major problem. To overcome this drawback, we incorporate LPAR Routing Framework which can further enhance the transmission quality.

Location based point to point adaptive routing (LPAR), each node can make routing decision based on node location, which means nodes are flexible to changing topology and reduce route recalculations at the source. Various network parameters like throughput, packet delivery ratio, end to end delay, packet loss etc. are estimated to analyze the service quality.

\section{SYSTEM IMPLEMENTATION METHODOLOGY}

For the purpose of testing the accuracy and assessing the effectiveness of the proposed technique of incorporation of LPAR Routing Framework with Conventional Routing networks In ODMRP, the said system is designed and implemented for the purpose of simulation. The simulation is carried out with the help of MATLAB software package. The implementation method as well as the results obtained from the same are discussed in subsequent section.

\subsection{Implementation Method}

Implementation Process for Optimization Algorithms like Hybrid Ant Colony Optimization Algorithm (ACA) and Improved Genetic Algorithm is explained in the subsequent paragraph.

The primary step for Hybrid Ant Colony Optimization Algorithm (ACA) is the Generation of Random values Based. The random values so generated have to be based on the constraints of the bandwidth limits. Thus, the random generation is dependent upon the minimum and maximum values of bandwidth of WSN.

For the same purpose, the program implements the following Equation in MATLAB code.

$$
I=\min \_v a l+\left(\max \_v a l-\min \_v a l\right) * \text { rand }
$$

The equation above gives the initial size (I) which is dependent upon the population size described in paper. Further, the program is designed in such a way that all the above values have to be initially passed on to the objective function for further process. Note that *objective function: throughput, packet delivery ratio, end to end delay, packet loss along with optimal routing *. After the above steps are thoroughly done, the selection of a unique best value from the fitness function has to be made. Following which, the initial random values are updated with the help of integration of the algorithm parameters. The program proceeds and repeats the above step of selection of an optimum value. This process is repeated for times equal to the number of iterations specified by the user. At the end of the optimization process, the program displays the output and compares it with the results obtained by existing techniques as well as other optimization algorithms.

To undertake the said simulation, a single program is built after combining various smaller MATLAB programs which were coded to represent the functions. The MATLAB function program developed which collectively work for the said technique are CSMA.m, CSMACA.m, friis.m, IPv6.m, LinkModel.m, macmodel.m, manet.m, mobility.m, ODMRP.m and TDA.m.

The output at the end of each process of optimization gives the values for the following:

1. Overall Efficiency of the network achieved by using each method

2. The Error value

3. Parameters like End to End delay, Packet loss and throughput of the network

\section{DISCUSSION OF RESULTS}

The program designed in the MATLAB software for the purpose of incorporation of LPAR Routing Framework with Conventional Routing networks In ODMRP, shows the following results.

Firstly, on starting the simulation, the topology of a 10 node network would be built which would be similar to the one shown above in figure 1 . At a subsequent stage, the nodes start to communicate with each other. From figure 1 It can be seen that node 6 and node 4 are in the process of 
communication wherein, a vector or link can be seen between these nodes. With the passage of time and further progress in the simulation, all the nodes would be connected in the communication process and finally one can see the network mapped with nodes and communication links.

\subsection{0 node network:}

Figure 1. 10 node network at the start of the process of communication

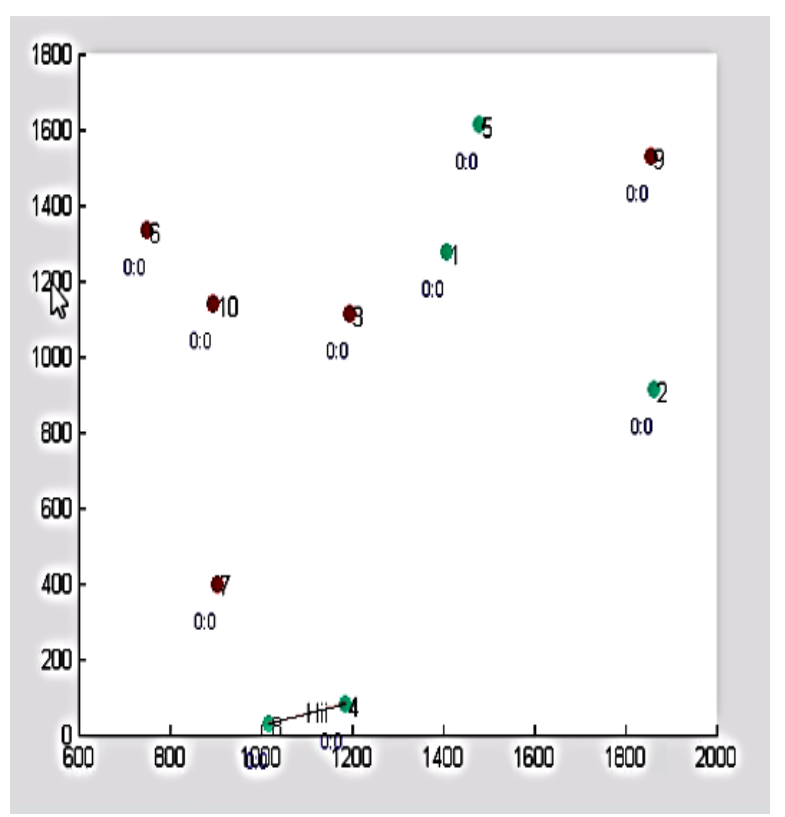

Finally, the result of the optimization with the said technique is seen in figure 2 . Here, the algorithm is repeated for a fixed number of times for the 10 node system. The graph shows the best value vs. number of iterations. From the figure 2 it can be seen that the resultant value initially oscillates but soon converges to the best value in and after that the value remains constant in spite of the process repeating numerous times

Figure 2: Result of ant colony optimization

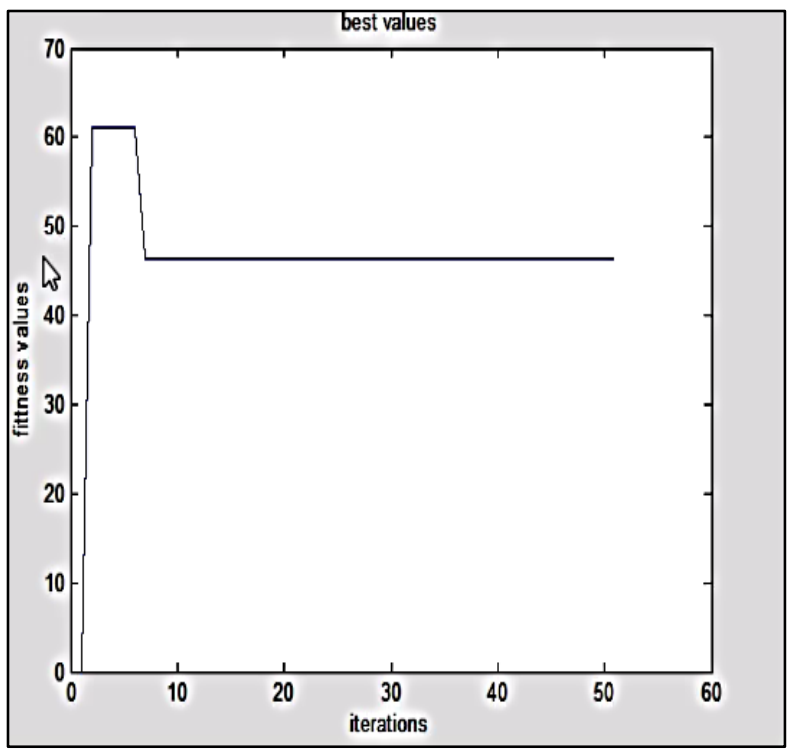

\subsection{0 node network:}

Figure 3: 50 node network topology

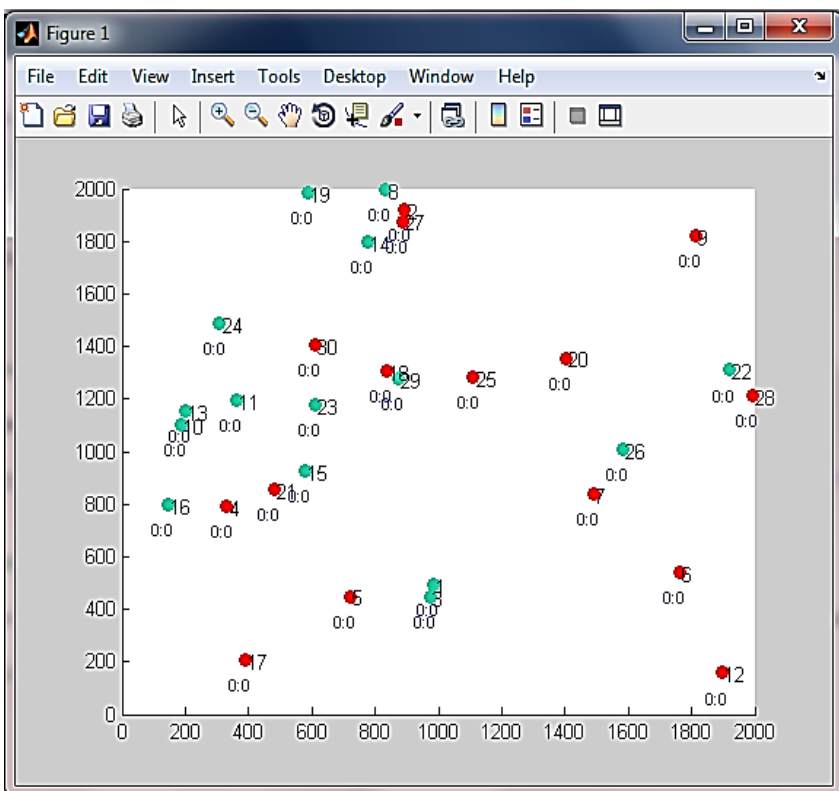

To check the scalability of the network, the same program implements a 50 node network. Similar o the process described in the 10 node network;

Figure 4: 50 node network at the start of the process of communication

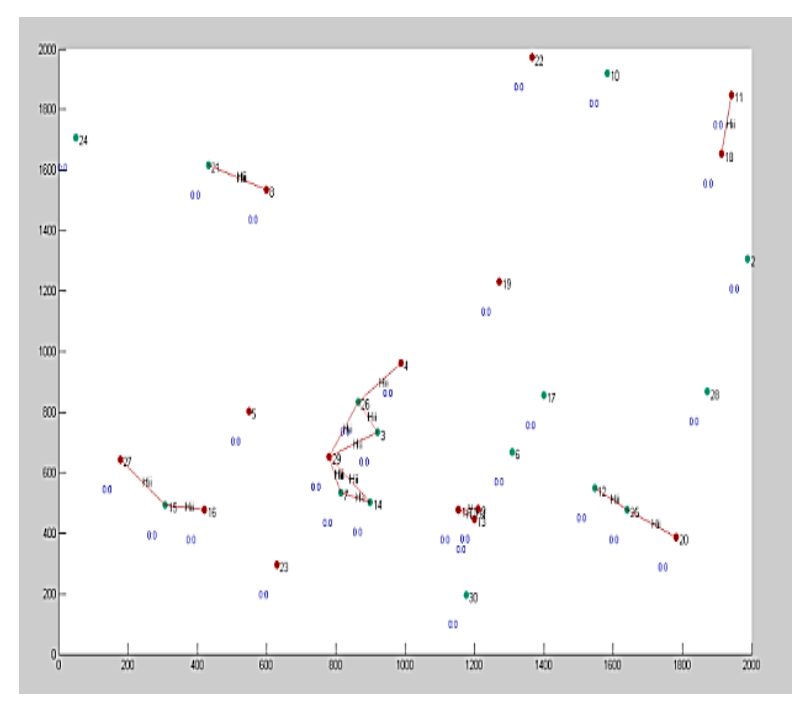

The map of the network with all nodes communicating can be seen in the figure 5

Initially, on starting the simulation, the topology of a 10 node network would be built which would be similar to the one shown above in figure 3 . At a subsequent stage, the nodes start to communicate with each other. From figure4.it can be seen that various nodes are in the process of communication wherein, a vector or link can be seen between these nodes. With the passage of time and further progress in the simulation, all the nodes would be connected in the communication process and finally one can see the network mapped with nodes and communication links. 
Figure 5: 50 node network mapping all communications

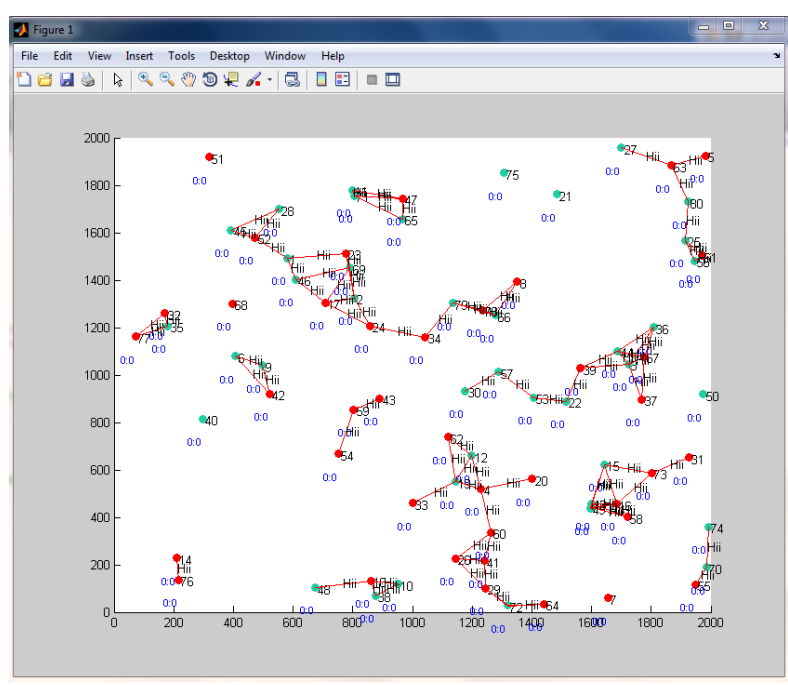

Finally, the result of the optimization with the said technique is seen in figure 6. Here, the algorithm is repeated for a fixed number of times. The graph shows the best value vs. number of iterations. From the figure it can be seen that the result converges to the best value in the initial stage itself after which the value remains constant in spite of the process repeating many times.

Figure 6: Result of any colony Optimization

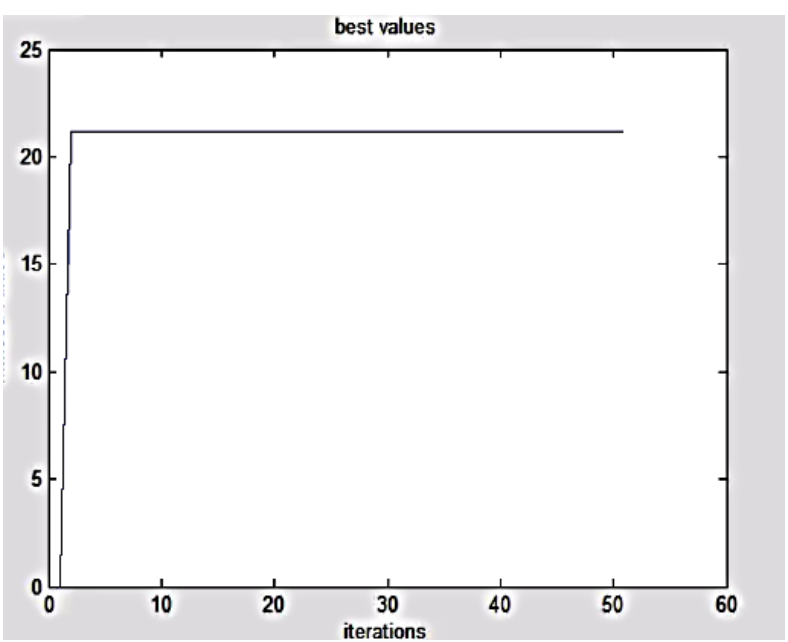

Hence, the proposed system performs effectively for the optimization of MANET and undertakes the process of routing efficiently. The scalability of the system is proved with the help of 50 node system which has more complexity in the communication links and the map for the same is shown.

\section{CONCLUSION}

This paper has undertaken an in-depth study of the available methods of mobile adhoc network routing and proposes a unique technique for Incorporation of LPAR Routing Framework with Conventional Routing networks In ODMRP. Here, the said technique is implemented using the Cross-Layered Ant Colony Optimization Multicast Routing Protocol which is discussed in detail in the preceding sections. The study undertakes simulation for the same with the help of mobile networks which are initially 10 nodes and checks the scalability up to a total of 50 nodes.

The simulation results when Cross-Layered Ant Colony Optimization Multicast Routing Protocol was implemented using MATLAB have been discussed. The results clearly indicate that the said technique performs efficiently giving effective solution for reduction of congestion overhead and security in a mobile adhoc network undertaking multicast.

\section{REFERENCES}

[1] Abolhasan, M., Wysocki, T. A., \&Dutkiewicz, E. (2003). LPAR: an adaptive routing strategy for MANETs. Journal of Telecommunications and Information Technology, 28-37.

[2] Aggelou, G., \&Tafazolli, R. (1999, August). RDMAR: A bandwidth-efficient routing protocol for mobile adhoc networks. In Proceedings of the 2nd ACM international workshop on Wireless mobile multimedia (pp. 26-33).ACM.

[3] Boppana, R. V., \&Konduru, S. P. (2001).An adaptive distance vector routing algorithm for mobile, adhocnetworks.In INFOCOM 2001.Twentieth Annual Joint Conference of the IEEE Computer and Communications Societies.Proceedings.IEEE (Vol. 3, pp. 1753-1762).IEEE.

[4] Chlamtac, I., Conti, M., \& Liu, J. J. N. (2003). Mobile adhoc networking: imperatives and challenges. Adhoc networks, 1(1), 13-64.

[5] Goldsmith, A. J., \& Wicker, S. B. (2002). Design challenges for energy-constrained adhoc wireless networks. IEEE wireless communications, 9(4), 8-27.

[6] Jetcheva, J. G., \& Johnson, D. B. (2001, October).Adaptive demand-driven multicast routing in multi-hop wireless adhocnetworks.In Proceedings of the 2nd ACM international symposium on Mobile adhoc networking \& computing (pp. 33-44).ACM.

[7] Karyotis, V., Stai, E., \& Papavassiliou, S. (2013). Evolutionary Dynamics of Complex Communications Networks.CRC Press.

[8] Ko, Y. B., \& Vaidya, N. H. (2000). Location-aided routing (LAR) in mobile adhoc networks. Wireless networks, 6(4), 307-321.

[9] S.A. Ade, P.A. Tijare "Performance Comparison of AODV, DSDV, OLSR and DSR Routing Protocols in Mobile Ad Hoc Networks", International Journal of Information Technology and Knowledge Management. July-December 2010. Volume 2 pp. $545-548$

[10] P. Venkata Krishna and Iyengar, "A Cross Layer Based Qos Model For Wireless And Mobile Networks" Journal of Mobile Communication1 (4): 114-120, 2007 